\title{
Preface
}

\section{Contemporary Approach to Lower Extremity Reconstruction}

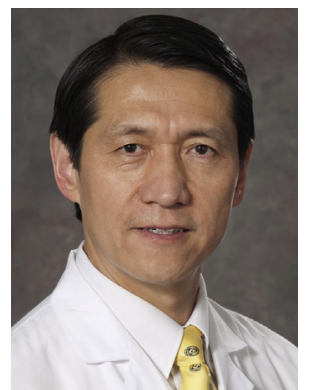

Lee L.Q. Pu, MD, PhD, FACS, FICS

Editor

Lower extremity reconstruction, as a subspecialty of plastic surgery, is an important part of the reconstructive surgery we do as plastic surgeons. For the past 20 years, many innovative techniques for lower extremity reconstruction have been discovered, for example, advanced wound management and microvascular free-tissue transfer, and development of new perforator-based flaps for lower extremity reconstruction. At many tertiary medical centers in developed countries, lower extremity reconstruction has become more than just soft tissue coverage, since functional restoration has also been emphasized through a multidisciplinary approach. It is important for reconstructive plastic surgeons to be familiar with the most recent innovations in lower extremity reconstruction so that the best possible reconstructive outcome can be provided to their patients.

As an active member of both American Society for Reconstructive Microsurgery and World Society for Reconstructive Microsurgery, I believe one of my primary responsibilities is to promote scientific exchange for reconstructive plastic surgery. For this reason, I decided to accept another invitation by Elsevier to edit a new issue of Clinics in Plastic Surgery on lower extremity reconstruction. I have chosen this topic because of my ongoing clinical experience on lower extremity reconstruction and expertise on editing a textbook for lower extremity reconstruction. The goal of this new issue in the Clinics in Plastic
Surgery is to provide readers a precise but comprehensive overview on the contemporary approach to lower extremity reconstruction. For this reason, I have invited several contributors who not only are renowned experts in the field but also have published extensively in lower extremity reconstruction.

In this 19-article issue, the first 4 articles focus on refined or newly developed locoregional flaps, such as hemisoleus muscle flaps, distally based sural artery flaps, propeller flaps, perforator-plus flaps, and freestyle local perforator flaps for soft tissue reconstruction of the lower extremity. The next 5 articles focus on perforator-based microvascular free tissue transfer, including some new workhorse flaps, such as the medial sural artery perforator flap, the superficial circumflex artery perforator flap, and freestyle free perforator flaps. This is followed by another 9 articles, and each focuses on a unique issue in lower extremity reconstruction, such as the optimal timing for traumatic lower extremity reconstruction, management of Gustilo type IIIC injuries, orthoplastic reconstruction, functional restoration, super-microsurgery, lower extremity reconstruction after sarcoma resection, free tissue transfer for chronic wounds, and lower extremity reconstruction for both pediatric and elderly populations. The issue ends with a special article on the combat experience for limb salvage and functional restoration.

As guest editor, I sincerely hope that you enjoy reading this special issue of Clinics in Plastic 
Surgery and find it useful for your busy practice. It represents a true team effort from many worldrenowned expert surgeons from United States, South Korea, Taiwan, and Switzerland. I would like to express my heartfelt gratitude to all contributors for their expertise, dedication, and responsibility to produce such a world-class issue of plastic surgery. It is certainly my privilege to work with these respected authors in this important field of plastic surgery. I would also like to express my special appreciation to the publication team of Elsevier, who has put this remarkable issue together with the highest possible standard.

Lee L.Q. Pu, MD, PhD, FACS, FICS Division of Plastic Surgery

Department of Surgery University of California, Davis 2335 Stockton Boulevard, Room 6008 Sacramento, CA, 95817, USA

E-mail address: Ilpu@ucdavis.edu 